\title{
The hazards of government data
}

Science thrives in a culture of accessibility and transparency that government often finds disadvantageous. Controversies in BSE research and environmental protection illustrate the dangers of secrecy.

WHENEVER scientific research lies close to the sharp end of political debate, the issue of who has access to data takes on a critical importance. If it is governments that control this access, the potential conflict over who best represents the 'public interest' is clear. In such circumstances, researchers in government laboratories are central players, and can therefore be torn, being required to meet the interest of their employers on the one hand and of the wider research community on the other. Current debates in the United States and Europe illustrate how the second of these roles can be all too easily undermined.

Any government agency that collects information with even a potential bearing on policy has a basic responsibility to ensure that such information is preserved for future reference. That statement would have seemed unnecessary were it not for the remarkable action taken last April by the California Environmental Protection Agency (CEPA), instructing scientists in its Office of Environmental Health Hazard Assessment to "dispose of all documents... and other communications prepared during the course of policy formulation which contain other policy proposals not adopted or reflected in the finals decision". Disingenuously claiming that its policy was intended to encourage scientists to provide advice without fear of their views becoming known, the agency has now, in the light of protests and court challenges, been ordered by the state government to reverse the instruction (see page 470).

Yet, while almost all government agencies would accept that the need to preserve their stocks of information is self-evident, another seemingly innocuous responsibility can present them with greater difficulty: maintaining such information in a form that is accessible and comprehensible to anybody who may have a subsequent interest in it. Government departments can be abolished and responsibilities transferred, and orderly documentation of files may get disrupted or even destroyed in the process. Such appears to have happened in the United Kingdom, where the abolition of the Milk Marketing Board has made it more difficult for data on dairy herd demography to be obtained, thus obstructing studies of bovine spongiform encephalopathy (BSE).

Even less acceptable is the situation in which sheer lack of resources appears to leave bodies unable to cope fully with their responsibilities. That is said by researchers to be a severe problem at the UK Central Veterinary Laboratory, which plays a critical role in the provision of BSE data - a problem denied by its owner, the Ministry of Agriculture, Fisheries and Food (MAFF) (see page 467).

Keeping documents stored and filed is essentially a duty of care. A further role of government agencies - that of making data available to others - can be more contentious. The CEPA, for example, is now indefensibly seeking to keep its stock of information, saved from destruction, under lock and key. In Europe, a duty of accessibility is made explicit in the case of environmental regulatory bodies that, according to a European directive, are obliged to make available to the public any data that they have collected. But, in practice, activists have found that the lack of a clear definition of which bodies are covered, and the expense of pursuing judicial reviews of refusals of access, can allow some agencies to wriggle out of their responsibilities. Despite such weaknesses, the European directive provides a welcome boost to openness. Regrettably, it does not apply to non-environmental institutions such as MAFF and its laboratories and their continental equivalents.

Even in countries where freedom of information or 'open government' policies are practised, a desire to protect the interests of suppliers of information - such as businesses, farmers or patients - can allow data to be exempted from the requirement that it is made openly available. But such protection of special interests can often collide with pressures for analyses of a controversial issue by researchers who are independent of government.

The political use of science in the BSE saga highlights the need for such independent analysis as much as any other issue. But even nongovernment scientists argue that access to data should be restricted to those sufficiently sophisticated to interpret its significance responsibly. In particular, they worry about how the mass media or even other scientists can be trusted with data that require a sensitive approach to understand all the necessary caveats. Such concern, for example, lies behind restricted access granted in the United Kingdom to databases of suspected cases of the new variant of Creutzfeldt-Jakob disease, as well as to data on BSE infection. The latter data have often been made available only after strenuous efforts to obtain them - in other words, when the political pressure on MAFF has proved irresistible.

The contrast of that approach to the openness involving data on the AIDS epidemic is striking, while its poor consequences for science are all too obvious. Only one group of nongovernment scientists has had the belated opportunity to conduct an analysis of BSE epidemiology in the United Kingdom. As it happens, that paper (R. M. Anderson et al. Nature 382, 779-788; 1996) incorporates results from a separate study of data concurrently submitted to Nature that has been publicized as demonstrating maternal transmission of BSE. The latter paper has since been withdrawn, and its senior author, John Wilesmith, is reported to have told a meeting last week that it is now impossible to be sure of the extent to which maternal transmission might be occurring, if at all (The Veterinary Record 139, 328-329; 1996). The algorithm of Anderson et al. remains unaffected but, pending further analysis, those seeking to draw policy conclusions from it have an even more uncertain basis than before. Undoubtedly, everybody would be better placed if MAFF had been more open with its data in the past - not only with outside researchers, but also with other government departments.

In short, recent events support a belief that government agencies can, all too often, compromise their commitment to open communication - even between scientific experts - in order to preserve their own interests and those of their 'client' groups. Yet where issues of environmental and health protection are concerned, the need, if only in the cause of good science, to balance this with an equally strong need to meet the interests of the broader community suggests that the case for open access has never been more strong - or more urgent. CEPA's secrecy is being publicly opposed. Who will tackle MAFF? 